\title{
BMJ Open Impact of multimorbidity on healthcare professional task shifting potential in patients with type 2 diabetes in primary care: a French cross-sectional study
}

Irène Supper, ${ }^{1,2}$ Yann Bourgueil, ${ }^{3}$ René Ecochard, ${ }^{4}$ Laurent Letrilliart ${ }^{1,2}$

To cite: Supper I, Bourgueil Y, Ecochard R, et al. Impact of multimorbidity on healthcare professional task shifting potential in patients with type 2 diabetes in primary care: a French crosssectional study. BMJ Open 2017;7:e016545. doi:10.1136/ bmjopen-2017-016545

- Prepublication history and additional material for this paper are available online. To view these files, please visit the journal online (http://dx.doi. org/10.1136/bmjopen-2017016545).

Received 21 February 2017 Revised 13 July 2017 Accepted 21 July 2017

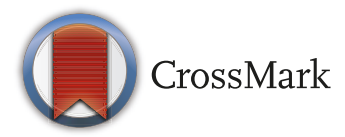

${ }^{1}$ Department of General Practice, Université Claude Bernard Lyon 1, F-69008 Lyon, France

${ }^{2}$ Health services and performance research, Univ. Lyon, Université Claude Bernard Lyon 1, HESPER EA 7425, F69008 Lyon, France

${ }^{3}$ IRDES, Institute for Research and Information in Health Economics, F-75019 Paris, France

${ }^{4}$ Department of Biostatistics, Hospices Civils de Lyon, Lyon, France

Correspondence to

Dr Irène Supper;

irenesupper@hotmail.com

\section{ABSTRACT}

Objectives To estimate the transferability of processes of care from general practitioners (GPs) to allied healthcare professionals and the determinants of such transferability. Design French national cross-sectional multicentre study Setting 128 family practices providing supervised training for residents in general practice.

Participants All patients consulting with their GP over a total number of 20 days (ie, 1 day a week from December 2011 to April 2012). Encounters where type 2 diabetes was one of the managed health problems were selected for analysis.

Primary and secondary outcome measures Processes that were associated with specific health problems were collected by 54 residents. Potential process transferability was the main outcome assessed, as well as the professionals involved in the collaboration and the eventual conditions associated with transfer.

Results From 8572 processes of care that concerned 1088 encounters of patients with diabetes, $21.9 \%$ (95\% Cl $21.1 \%$ to $22.8 \%$ ) were considered eligible for transfer from GPs to allied healthcare professionals $(78.1 \%$ to nurses, $36.7 \%$ to pharmacists). Processes were transferable with condition(s) for $70.6 \%$ (ie, a protocol, shared record or supervision). The most transferable processes concerned health maintenance (32.1\%) and cardiovascular risk factors (hypertension (28.7\%), dyslipidaemia (25.3\%) and diabetes (24.3\%)). Multivariate analysis showed that educational processes or a long-term condition status were associated with increased transferability (OR 3.26 and 1.47, respectively), whereas patients with higher intellectual occupations or those with two or more associated health problems were associated with lower transferability (OR 0.33 and 0.81 , respectively). Conclusions A significant part of GP activity relating to patients with multimorbidity including type 2 diabetes could be transferred to allied healthcare professionals, mainly on prevention and global education to cardiovascular risk factors. The organisational and finance conditions of team work as views of patients and healthcare professionals must be explored before implementation in primary care.

\section{BACKGROUND}

Among other chronic diseases, the worldwide prevalence of type 2 diabetes is expected
Strengths and limitations of this study

- Multicentre national study

- A large sample of 1088 diabetes patient-physician encounters from 128 representative GP practices

- A practice-based analysis of health problems and associated processes of care

- Contextualised practice-based data regarding process of care transferability

- Subjectivity of the judgement of transferability by residents

to increase from 171 million in 2000 to 366 million in 2030. ${ }^{12}$ As an independent risk factor, diabetes doubles the risk of cardiovascular disease. ${ }^{3}$ To tackle the epidemiological transition from acute to chronic conditions such as diabetes, health policymakers have considered the merits of changing the skill mix in primary care by delegating or transferring certain tasks from general practitioners (GPs) to other primary healthcare professionals. ${ }^{4}$ Such an approach is expected to address workforce shortages as well as help to meet overwhelming demands for highquality affordable care. ${ }^{4}$ Presently, task delegation or transfer tends to evolve towards different forms of skill mix (changing role as substitution, delegation, enhancement or introduction or changing interfaces between services as transfer, relocation, liaison ${ }^{4}$ and more broadly interprofessional collaboration). ${ }^{5}$ In Europe, interprofessionnal collaboration is defined as cooperation among different healthcare professionals, integrating complementary competences and skills to make the best possible use of resources. ${ }^{5}$

Delegation of certain tasks that were previously performed by GPs to nurses has been associated with an improvement in short-term surrogate endpoints in patients with type 2 diabetes. ${ }^{67}$ In primary care in France, the delegation of diabetes follow-up and therapeutic 
education to practice nurses led to improved $\mathrm{HbA}_{1 \mathrm{C}}$ levels in patients. ${ }^{8}$ In the Netherlands, delegation of routine diabetes care to practice nurses combined with computerised decision support and performance feedback to GPs reduced cardiovascular risk but did not improve $\mathrm{HbA}_{1 \mathrm{C}}$ levels. ${ }^{9}$ In a 2010 review that was focused mainly on the UK, the use of nurses to partly replace GPs in providing diabetes care was shown to have a positive impact on glycaemic control in patients with diabetes. ${ }^{10}$ The pharmaceutical care model, an alternative method of diabetes management whereby all information relating to diabetes management is provided exclusively by a pharmacist, has been tested but without long-term in-depth evaluation. ${ }^{10} 11$

Quality improvement strategies that target health systems have a higher impact on $\mathrm{HbA}_{1 \mathrm{C}}$ levels than strategies that focus on individual professionals. ${ }^{12}$ Multidisciplinary approaches for team changes, including the expansion or revision of professional roles (eg, a more active role for nurses or pharmacists in monitoring patients or adjusting drug regimens) have been shown to improve glycaemic control, low-density lipoprotein levels, blood pressure and weight. ${ }^{12-14}$ In North America, multidisciplinary teams involved in a physical activity self-management programme for primary care patients with multimorbidity and diabetes were associated with a reduction in $\mathrm{HbA}_{1 \mathrm{c}}$, weight or frailty. ${ }^{15}$ However, high-quality evidence on long-term morbimortality outcomes is still needed. Task delegation for patients with diabetes is already widely implemented in several countries, including the USA and the UK, but less widely in France and Germany. In France, primary care is traditionally characterised by the predominance of private practices for GPs as for allied professions, which all operate under a fee-for-service system. Patients pay a fee to professionals and are then reimbursed by the mandatory national health coverage and complementary insurances. Those with longterm conditions or low incomes or migrants benefit from a fee exemption status. Historically established in solo practices, the majority of GPs now work in small groups. ${ }^{16}$ Some of them (around 10\%) tend to engage in interprofessional group practices which support team work and skill mix innovation, with public policy support, while in other countries such as Canada, interprofessional group practices have already become generalised as the gold standard of care. ${ }^{17}$ Nevertheless, the scalability and modalities of task delegation in common primary care practices in the French context (ie, different primary care professionals in different locations), including the multimorbidity of patients with chronic disease, need to be assessed. ${ }^{18}$

The issue of task delegation can be considered at both the patient and practice levels. At the patient level, task delegation must be individually tailored due to the fact that $40-90 \%$ of patients have multimorbidity in routine primary care encounters. ${ }^{19-21}$ Multimorbidity is defined as any combination of chronic disease with at least one other disease (acute or chronic) or biopsychosocial factor (associated or not) or somatic risk factor. ${ }^{22-24}$ To address the complexity associated with multimorbidity in primary care, it is necessary to focus on the health problems that are relevant at the point of $\operatorname{care}^{212425}$ - that is, those health problems that influence clinical management.

At the practice level there is a lack of evidence regarding the needs of individual patients and, consequently, the most adapted skills in response to these needs. The means of combining various team changes (expansion or revision of professional roles) are yet to be studied. ${ }^{12}$

A practice-based research approach would seem to be necessary, including a reflection on the appropriateness of transferring the various tasks previously undertaken by GPs to allied healthcare professionals. The aim of this study was to investigate the transferability of healthcare tasks (or processes of care), from the perspective of GPs, from GPs to allied healthcare professionals, and to identify the factors that determine that transferability.

\section{METHODS}

This study of patients with type 2 diabetes ('diabetes') formed part of the French multicentre ECOGEN study (Etude des Eléments de COnsultation en médecine GENérale), which was conducted from December 2011 to April 2012. Details of the cross-sectional ECOGEN study design have been published previously. ${ }^{26}$ The ECOGEN study was conducted in a nationally representative sample of 128 GP practices across France, where participants received their usual care from their GP. The study investigators were 54 voluntary GP residents (ie, trainees specialising in family practice under direct supervision), who observed the consultation and collected data on the usual care provided by their 128 GP trainers. Each resident was placed in two or three different practices and no practice had more than one resident.

Within each practice, all consecutive patients seeking care, including children and adults, were recruited to participate in the ECOGEN study. The ECOGEN database only recorded encounters and any related health problems managed, provided that the patient encounter involved its management through at least one process of care. Patient morbidity which did not result in any process of care during the encounter (past medical history or health problems not related to the encounter) were not recorded. During data analysis we selected patients from the ECOGEN database who had a diagnosis of type 2 diabetes for inclusion in this nested study. A total of $168(0.8 \%)$ patients declined to participate in the ECOGEN study due to the requirement that a trainee doctor be present during the consultation. During a 2-day seminar the study investigators (ie, resident trainees) were trained to collect and code structured consultation data according to the requirements of the International Classification of Primary Care (ICPC-2) to analyse the potential for task delegation and to record data in a central database using a digital encoding engine. ${ }^{27}$ The five types of processes were secondarily binary recoded as preventive versus non-preventive processes of care.

\section{Data collection and recording}

The ECOGEN study investigators directly observed 20613 patient-GP encounters ('encounters'), regardless of the 
reasons for each encounter, over a total number of 20 days during the study period (ie, 1 day a week over 5 months). The processes of care ('processes') were all tasks carried out by the GP relating to the health problems that were managed during the encounters. The term 'process of care' includes preventive, diagnostic, curative, administrative and coordinative tasks, referred to as processes or procedures in the ICPC-2 (see online Supplementary appendix 1). ${ }^{27}$ Health problems, which were assessed as being either a symptom or a diagnosed health issue, were recorded in the study if they generated at least one process of care. Study investigators collected data relating to individual encounters (place, date and reasons for the encounter, diagnostic assessment, processes), individual patient characteristics (gender, age, existing or new patient, profession, fee-exemption status) and individual GP characteristics (age, gender, solo or group practice, location, number of annual patient encounters, use of fixed fees vs variable fees). The ICPC-2 was used to classify the observed processes of care used in the encounter. ${ }^{27}$ The patient occupations were recorded according to the French classification of Occupations and Socio-occupational Categories described by the National Institute of Statistics and Economic Studies. ${ }^{28}$ Categories are: (1) farmers; (2) tradesmen, shopkeepers and heads of businesses; (3) managers and higher intellectual professions; (4) associate professionals; (5) services, sales and administrative employees; (6) workers; (7) retired; (8) others not in work. Category (3) encompasses managers and higher intellectual professions (ie, self-employed professionals and allied: civil service managers, intellectual and artistic professions, managers in businesses).

A record was made of patients who had medical fee exemption status that covered long-term health conditions or low income status. The term 'multimorbidity' is used in this study to denote the numerous health problems managed during the encounters including chronic, acute and preventive situations. This definition differs from the EGPRN definition as we refer to health problems managed during the consultation and not all patients' health problems. ${ }^{22}$ The study investigators evaluated the potential transferability of each process of care from the physician to an allied healthcare professional, where transferability of a process of care is deemed to be whether it is possible for it to be performed by a healthcare professional other than a GP, either by delegation or substitution. Delegation is moving a task up or down a traditional monodisciplinary ladder, with shared responsibility. Substitution expands the breadth of a job, in particular by working across professional divides or exchanging one profession for another, including the acceptance of new responsibility. ${ }^{4}$ Transferability assessment was encoded as not transferable, transferable without conditions or transferable with conditions (ie, supervision by a GP and/or availability of shared medical support and/ or a predefined protocol or else (suggestion to specify in free text). Predefined protocols, at the initiative of professionals from the field, have to describe the special law dispensation for task delegation and the involved healthcare professionals, according to the current legislation. ${ }^{29}$ If the task was judged transferable by the investigators, they had to indicate the various potential professionals concerned: nurse, pharmacist, psychologist, secretary, physiotherapist, midwife, social worker, and or other professionals (specified verbatim). This assessment was based on the residents' judgement in the context of the specific encounter, while assuming an acceptable availability of trained professionals and that the legal requirements were fulfilled. The judgement of the residents was based on the level of complexity and the risk associated with each process.

\section{Analysis of data}

Study authors parsed and analysed the data on all encounters for patients with diabetes being managed by their GP. Transferability was assessed for each process of care (ie, task) and analysed according to health problems, processes and involved professionals. The unit of analysis was the process of care. We measured the mean transferability of the processes of care associated with each health problem managed, including diabetes and any comorbidity. It was not possible to link patient records across the various encounters because the unit of analysis was the processes of care of the encounter and not the patient. For univariate analysis, the transferability according to mean age, number of health problems managed and processes was analysed with a Student's t-test and the categorical variables were compared with a $\chi^{2}$ test. The generalised linear mixedmodel fit by maximum likelihood (Laplace approximation) was adjusted for the clustering of patients by resident. The transferability of processes formed the dependent dichotomous variable. Explanatory independent variables were introduced into the model according to a forward stepwise selection. Processes of care were categorised into the following five groups: preventive, diagnostic, curative, coordinative and administrative. No interaction was found between the variables in the multivariate model. Analyses were computed using SPSS 25 software for univariate analysis and R 3.3.2 software for mixed models. This study received ethical approval and associated registration numbers from both the National Committee on Informatics and Freedom (CNIL, no 1549782) and the Committee for the Protection of Persons (CPP, no L11-149). No patient informed consent was required by these committees.

\section{RESULTS}

Among the 20613 encounters recorded in the ECOGEN database, 1088 (5.3\%) included the management of patients with diabetes. There were 1088 encounters, 8572 processes of care and 4038 health problems. These 1088 encounters were conducted by 128 GPs who assessed 4038 managed health problems including diabetes (a mean of 3.7 different health problems managed per encounter). GPs performed or prescribed 8572 processes of care (7.9 processes per encounter). Among these processes, $37.0 \%$ were related to diabetes and $63.0 \%$ were related to comorbidities. These encounters concerned patients with a mean (SD) age of 68.3 (11.6) years (range $28-100$ years) 
in the following age categories: <45: $26(2.4 \%) ; 46-55$ : $125(11.5 \%)$; 56-65: $304(27.9 \%)$; 66-75: 306 (28.1\%); 76-85: $262(28.1 \%)$; >86: $65(6 \%)$. From these encounters, $52.8 \%$ were men, $98.4 \%$ were known by the physician, $81.4 \%$ benefited from a fee-exemption status due to suffering from a long-term condition and $2.6 \%$ due to low income; the encounters took place at the primary care practice in $88.8 \%$ of cases and at home for the remaining cases. The encounters had a mean (SD) duration of 19.1 (9.4) $\mathrm{min}$.

\section{Global transferability}

The mean potential transferability for all these processes of care was $21.9 \%$ (95\% CI 21.1\% to 22.8\%) for all health problems $(24.6 \%$ (95\% CI $23.1 \%$ to $26.1 \%$ ) for diabetes and $20.3 \%$ (95\% CI $19.3 \%$ to $21.4 \%$ ) for comorbidities). A subsample of 63 encounters (5.8\%) concerned diabetes without multimorbidity which included 226 (2.6\%) processes. Among these, the mean potential transferability was $25.2 \%$ (95\% CI $19.5 \%$ to $30.9 \%$ ).

\section{Transferability according to health problems}

Diabetes, together with the 10 most frequently associated health problems managed during the encounter, represented $64.5 \%$ of all health problems managed in the encounters. Processes associated with 'health maintenance/prevention' were potentially considered the processes most suitable for transfer $(32.1 \%)$ and included preventive care processes such as prescription or provision of immunisation and the prescription of cancer screening (see online Supplementary appendix 2). Processes associated with major cardiovascular risk factors followed, with a transferability of $28.7 \%$ for hypertension, $25.3 \%$ for lipid disorders and $24.3 \%$ for diabetes. These three risk factors represented $57.5 \%$ of the 4038 health problems that were managed and they accounted for $49.4 \%$ of all processes (table 1).

\section{Transferability according to process of care}

There were 8572 processes of care, of which 3176 were related only to type 2 diabetes and 5396 to the other health problems managed during the encounter. The 10 most frequently involved processes accounted for 8061 $(94.0 \%)$. The 1747 top 10 transferable processes represented $92.9 \%$ of all 1880 processes considered potentially transferable. Therapeutic counselling and health education were considered the potentially most transferable processes for both diabetes $(53.0 \%$ and $48.3 \%$, respectively) and other morbidities (38.8\% and 39.3\%, respectively). Blood and urine tests, referral to a physician or a complete medical examination were considered potentially transferable more frequently when they were related to diabetes than to comorbidity (table 2).

\section{Transferability according to health professional}

A total of $78.1 \%$ of all potentially transferable processes were considered transferable to nurses and $36.7 \%$ to pharmacists. In cases of diabetes and multimorbidity, therapeutic counselling and health education were the processes that were most frequently considered potentially transferable to nurses and pharmacists. Therapeutic counselling was most frequently considered potentially transferable to psychologists when related to comorbidity. Beyond the five most frequent processes (table 3), medication prescription $(20.1 \%$ ) and partial medical examination $(20.1 \%)$ related to diabetes were

Table 1 Ten most frequently managed health problems ranked by transferability of processes of care

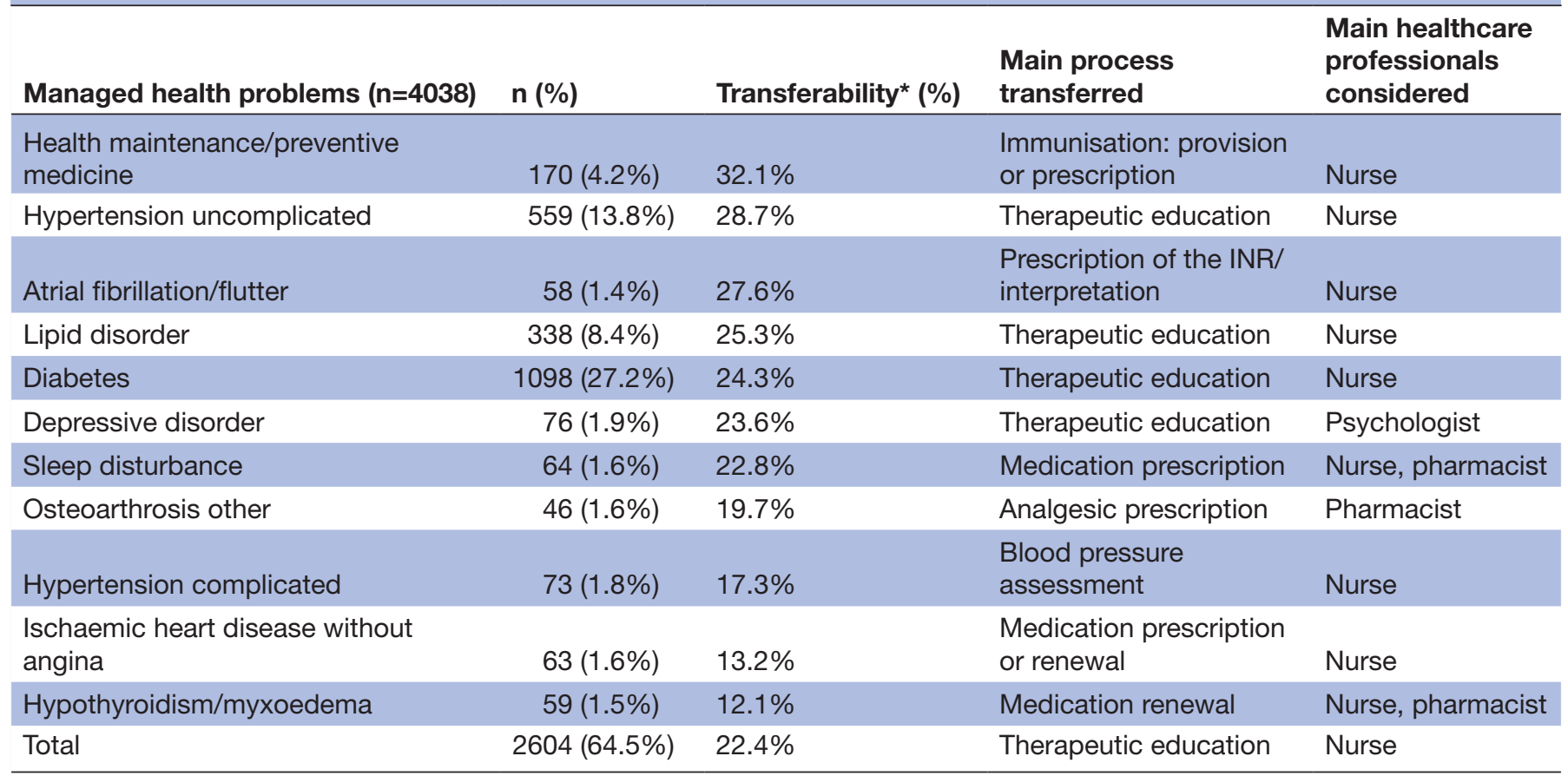

*Mean \% of transferability of the 8572 processes of care related to the specific health problem. 
Table 2 Ten most frequent processes of care ranked by the transferability of processes of care related to diabetes

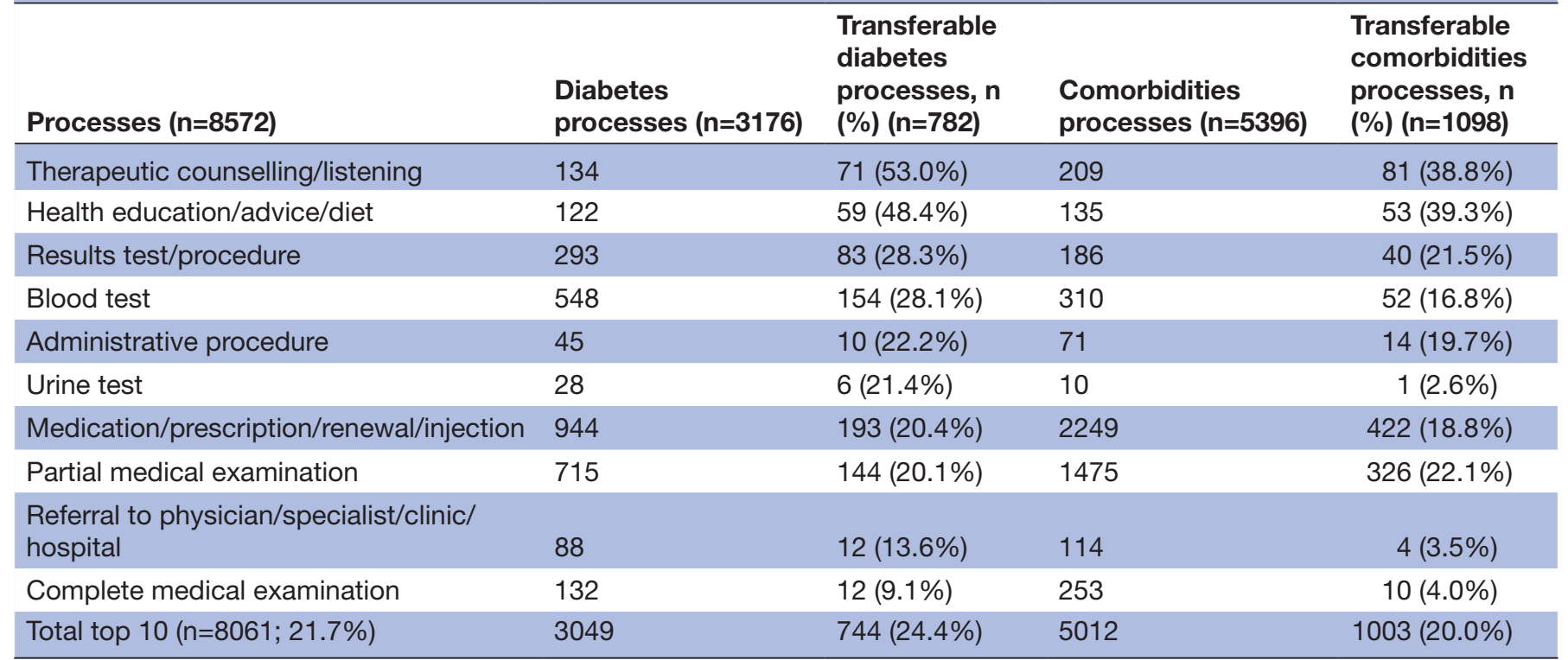

also considered potentially transferable, especially to pharmacists and nurses. In patients with atrial fibrillation, discussions of International Normalised Ratio results could be transferred mainly to nurses. Other professionals that were considered included psychologists for depressive disorders, especially for therapeutic counselling/listening, and pharmacists for sleep disturbance, osteoarthrosis and hypothyroidism, especially for medication prescription.

\section{Conditions for transferability}

Non-mutually exclusive practical conditions were required for $70.6 \%$ of potentially transferable processes (ie, $74.6 \%$ for diabetes and $67.8 \%$ for the comorbidities). These conditions were either a predefined protocol $(44.0 \%)$, a shared information system $(32.2 \%)$ or supervision by the GP $(31.7 \%)$.

The distribution of conditions was different between processes related to diabetes and to comorbidity $(\mathrm{p}<0.05)$. In particular, $52.7 \%$ of diabetes-related potentially transferable processes were under the condition of an existing protocol compared with $37.8 \%$ of processes related to comorbidity $\left(\mathrm{p}=3.1 \times 10^{-5}\right)$ (figure 1$)$.

\section{Determinants of transferability}

Multivariate analysis showed that determinants of potential process transferability that were specifically related to diabetes were affected by specific patient and encounter characteristics. Educational processes or suffering from a long-term health condition were both associated with an increased potential transferability (OR 3.26 and 1.47, respectively). Holding a higher intellectual profession or suffering from two or more associated health problems that were managed during the encounter were both associated with low potential transferability (OR 0.33 and 0.81 , respectively) (table 4 ).

\section{DISCUSSION}

From the 8572 processes of care that were performed by GPs in a patient consultation for diabetes, $21.9 \%$ of those processes were considered eligible for transfer from the GP to allied healthcare professionals. The most frequently occurring processes that were considered potentially most suitable for transfer concerned the following health problems: health maintenance/prevention $(32.1 \%$ of associated processes suitable for transfer), followed by processes associated with cardiovascular risk factors (processes associated with hypertension $(28.7 \%)$, lipid disorders $(25.3 \%)$ and diabetes $(24.3 \%))$. The health professionals to whom most processes could be transferred were nurses and pharmacists $(78.1 \%$ and $36.7 \%$ of the potentially transferable processes, respectively). A total of $70.6 \%$ of the potentially transferable processes could be transferable only under specific conditions, such as the existence of a predefined cooperative protocol, a shared medical record or supervision by the GP.

\section{Strengths and weaknesses of the study}

A strength of the ECOGEN study lies in its comprehensive dataset (only two missing data) of health problems managed in day-to-day practice with their associated processes, which included health education and coordination of care between healthcare professionals. The 128 GP trainers in this study were representative of GPs at the national level in terms of age, gender, type of contract with the social security system (fixed or variable fees), practice location (population size of the city) and annual number of encounters. In a previous study, French GP trainers were found to be representative of standard GPs in terms of patients and activities, ${ }^{30}$ therefore patients in our study can probably be considered representative of French patients. As no previous working experience in the interprofessional teams or 
with task delegation was required for the residents or for the GP trainers, transferability evaluation by investigators (ie, residents), who were specifically trained for the ECOGEN study, reflects their current views. This can be considered a weakness as residents might have had a limited knowledge of what the nurse's role can be in counselling and health education and a cautious view of task delegation or substitution. Conversely, it reflects the common perception of GPs and gives useful courses of action to implement skill mix in primary care with acceptability at a large scale, as it is just an emerging process in France. Future research should include consultation with interprofessional colleagues (ie, nurses, pharmacists) regarding their perspective on the transferability of processes of care.

The evaluation of process transferability was contextualised according to the profile of real consultation patients. Indeed, the residents took into account important characteristics of diabetic patients such as age, comorbidity and socioeconomic level rather than considering virtual diabetic patient categories. It was also contextualised to the actual organisation of GP practices, not only the $10 \%$ who are engaged in new models of multiprofessional health clinics. Of the 128 GP trainers, 70 double-checked the assessment of transferability in the main ECOGEN study for 1710 consultations, representing $8.2 \%$ of all consultations included in the database. This comparative evaluation of the process transferability showed no difference in the proportion of transferable processes and a fair agreement between residents and GP trainers in the main ECOGEN study (20.5\% vs $21.1 \%, \mathrm{p}=0.21 ; \kappa=0.37) .{ }^{31}$

Multivariate analysis enabled an investigation of the transferability based on the type of process of care and also on the characteristics of the encounter (number of combined health problems and patient characteristics). The risk of classification bias was limited by specific training of the residents to the ICPC-2 requirements and by the support of a digital encoding engine.

The implementation of task delegation in various healthcare systems brings the acceptability of task delegation by all professionals into question, although we could not assess such acceptability in this study. In the fragmented context of practice in primary care in France, as the various health professionals rarely work in the same location, skill mix is particularly difficult to implement without common tools (protocols, shared records). Our quantitative assessment of practical conditions (collaborative protocols, shared medical records or supervision) is complementary to nurses' views reported in other qualitative studies such as appropriate training and both recognition and respect of nurses' prerogatives and roles. ${ }^{32}$ In France the acceptability of task delegation from GPs to other healthcare professionals, regardless of the specific disease, ranges from $18.1 \%$ to $30 \% .^{31} 33$ Reflecting funding and payment issues in primary care where all providers are independent and paid by fee, the availability of public health insurance funding to support task delegation increases acceptability to $60 \%$. $^{31} 33$ 


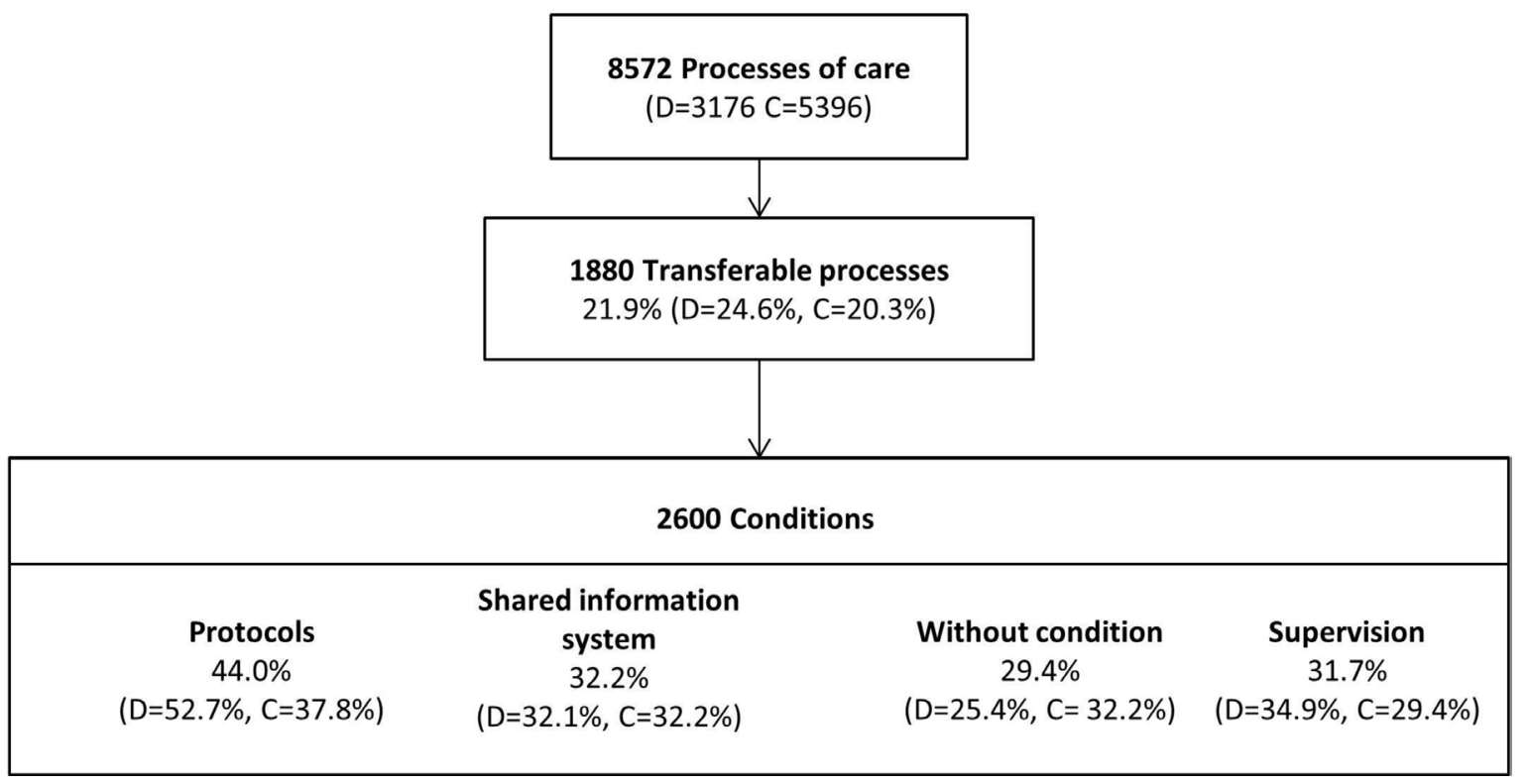

Figure 1 Conditions for potential transferability. C, comorbidity; D, diabetes.

\section{Educational processes as a priority}

According to our findings, the main factors allowing task delegation were less autonomous patients (with less intellectual occupations), a small number of health problems and especially with a need for preventive processes. Increased levels of complexity of patient encounters, as evaluated by the number of associated health problems (ie, the multimorbidity managed during each encounter) is associated with a decrease in the transferability of care processes. Only $2.1 \%$ of these encoded comorbidities were represented by diabetes-related complications (heart disease, stroke, retinopathy, nephropathy and diabetic foot). In France, $15 \%$ of diabetic patients were diagnosed with complications in $2009 .{ }^{34}$ From a primary care perspective, GPs tend to consider patients along with their individual multimorbidity. ${ }^{24}$ Major cardiovascular risk factors represented $57.7 \%$ of managed health problems that were associated with diabetes. We suggest, therefore, that the global cardiovascular risk should be addressed when collaborative actions are planned. ${ }^{35}$ Such an approach, which has been implemented for patients with diabetes based on the chronic care model, has been shown to decrease cardiovascular risk by $2.1 \%$ after 10 years. ${ }^{36}$ In practice, the estimation of cardiovascular risk, in particular by using a scoring system, is not yet an established process such that its transfer to nurses or pharmacists remains difficult due to complexity issues. ${ }^{37}$ Conversely, patient education of cardiovascular risk is a better defined task which looks more appropriate for task delegation. Diabetes was associated with multimorbidity in $44 \%$ of patients in an Irish study and in $90 \%$ in a retrospective Dutch study. ${ }^{19}{ }^{20}$ In our study, multimorbidity was associated with diabetes in $94.2 \%$ of encounters, likely because of our definition of multimorbidity and the presence of study investigators. Paradoxically, the absence of medical fee exemption status for a long-term condition was associated with lower transferability, probably because patients who did not qualify for medical fee exemption suffered from early or less severe forms of diabetes that required lower levels of intervention.

Higher intellectual professions were less targeted for transferability than any other professional category. Because of the low number of encounters, this result has to be confirmed. Nevertheless, social position is probably a proxy of health literacy and a greater capacity for self-management and, thus, a lower need for transfer. ${ }^{38}$ Indeed, the South Australian Healthplus trial found that, for at-risk patients (including diabetic patients), a key determinant of the need for coordination by nurses was a reduced capacity for patient self-management rather than illness severity. ${ }^{39}$

Transferability varies according to the type of process considered. Patient education processes had the strongest impact on transfer eligibility, consistent with results from the ongoing French ASALEE study (Action de Santé Libérale en Equipe - Health action by teams of self-employed health professionals), which is being implemented nationally using public experimental funds, with 360 nurses with advanced practices involved in pluriprofessional practices for diabetes and cardiovascular follow-up. 82940 Other results show that $31 \%$ of French GPs are in favour of transferring nutritional and therapeutic education, rising to $53 \%$ in cases where patient costs are met by the public health insurance system. ${ }^{33}$ The risk-benefit ratio of therapeutic education is low on all-cause mortality and morbidity criteria. ${ }^{41-44}$ Diabetes medications are associated with frequent and sometimes severe adverse events, such that the risk-benefit ratio is poorly understood without clear evidence of the effects of those medications on morbidity and mortality. ${ }^{45-47}$ Specific skills are needed to select and adapt the most appropriate treatment to the evolving patient statuses and scientific knowledge. Furthermore, 


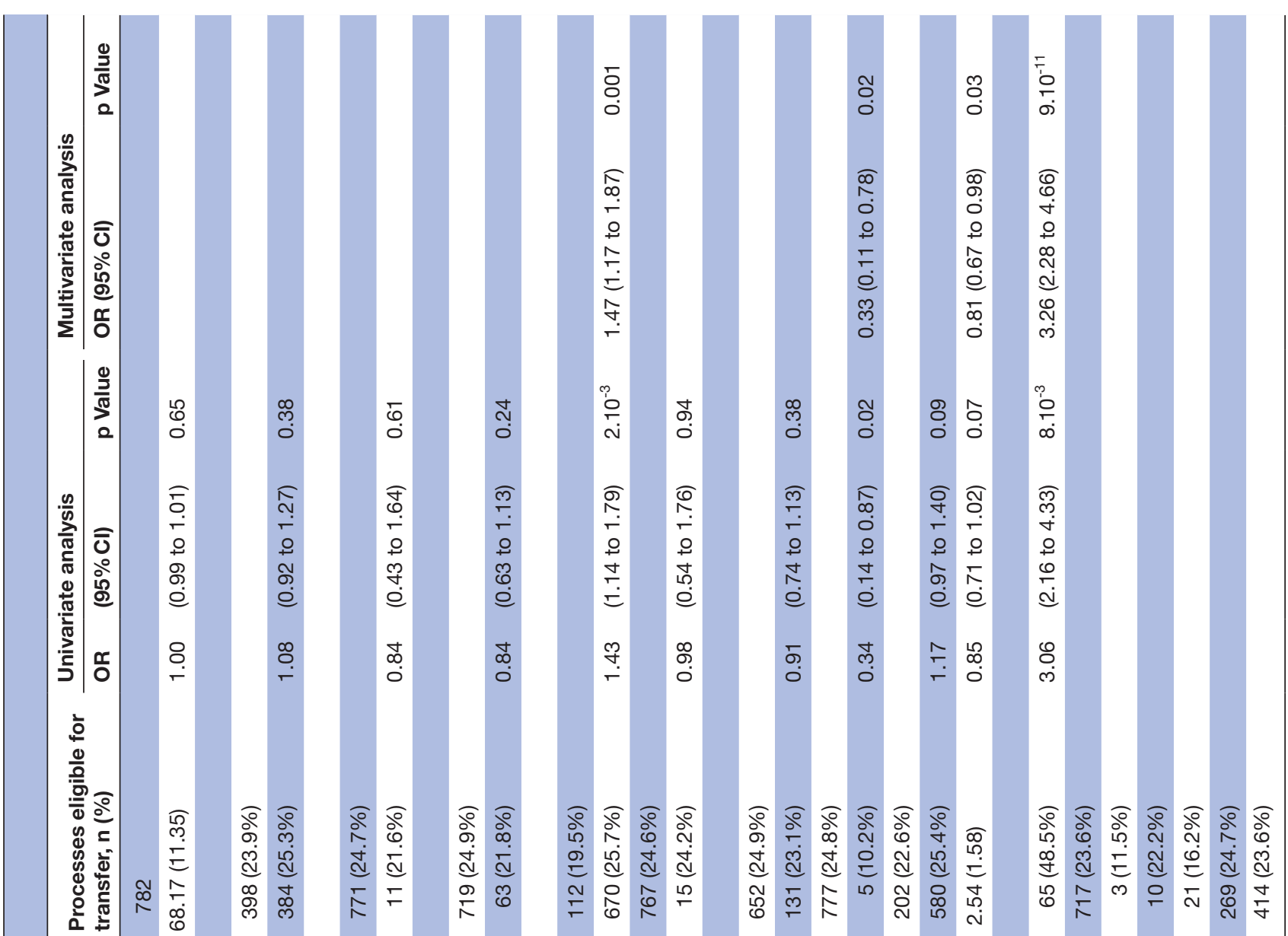

$\frac{\frac{0}{0}}{\frac{0}{0}}$

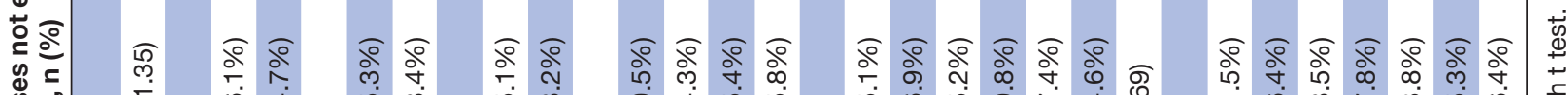

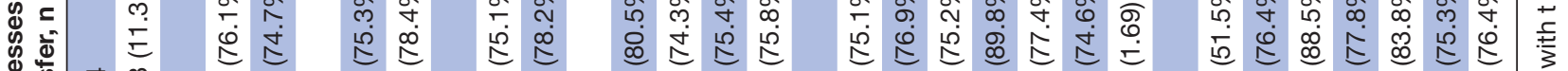

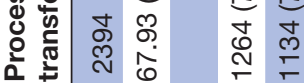

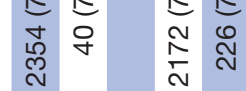

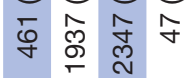

夏

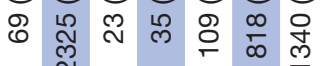

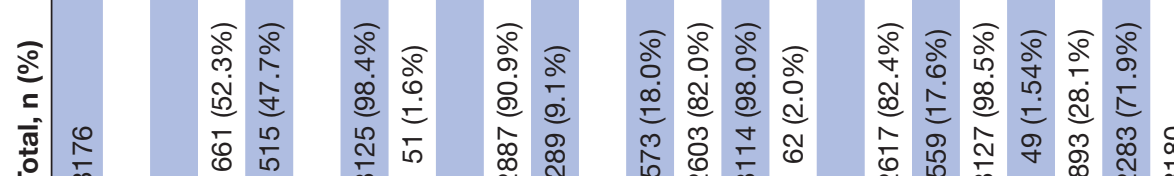

व ๙ิ)

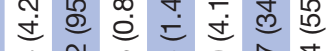


protocolising and transferring medication prescription in patients with diabetes is not yet widely generalised or accepted among healthcare practitioners, since such transfer requires appropriate training for medication prescription alongside critical decision making and independence from the pharmaceutical industry. ${ }^{48}$ Nurses are already charged with routinely prescribing diabetes medications in the USA, UK and Canada, ${ }^{48}{ }^{49}$ whereas in the Netherlands the transfer of this responsibility to practice nurses is still in the experimental stages. ${ }^{9}{ }^{50}$ In France, the current regulation allows the development of protocols including the transfer to nurses of medication prescription under medical supervision and responsibility. However, it is rarely implemented in primary care as team work is not developed in this setting.

\section{Implications for clinicians and public health}

The problem of fragmentation of disease management is well known. ${ }^{51}$ Interpersonal continuity of care for patients with diabetes may be disrupted by task delegation. ${ }^{2}{ }^{13}$ In addition, managing diabetes as part of a patient-centred approach requires integration over time of previous medical history, multimorbidity and the interaction between medical conditions and treatments. ${ }^{23} 5253$ Attention should be given to minimising the burden of follow-up by multiple professionals and multiple visits in patients with multimorbidity. ${ }^{52}$ The personal relationship with a single chosen GP results in improved patient satisfaction, improved compliance and reduced rates of hospitalisation and emergency room use. ${ }^{53} 54$ The relational continuity is inherent to the individualised treatment for education or depression. When transferring diabetes care from GPs to practice nurses in the Netherlands, some deterioration in patient health-related quality of life was observed, presumably because of the increased number and duration of visits with nurses and self-consciousness of illness. ${ }^{50}$ Conversely, collaborations with psychologists for patients with depression or anxiety within primary healthcare teams in Canada and Australia reduced health service use and costs, and improved patient quality of life. ${ }^{55}$ As a compromise between disease management and personal GP-patient relationships, the South Australian HealthPlus trial implemented a generic model of coordinated care for patients in primary care based on a sharing needs assessment and defined goals between patients and their caregivers. ${ }^{39}$ The loss of interpersonal and informational continuity requires specific support such as shared protocols, information systems and supervision. ${ }^{57}$

Consequently, future research regarding public health issues should study conditions of teamwork for optimal continuity and comprehensiveness and evaluation of criteria related to the global impact of task delegation. In particular, outcome criteria should include the overall number of visits, medication prescriptions ${ }^{58}$ and cost (for diabetes and all comorbidities), and steps in the healthcare pathway. Long-term clinical outcomes are also required, including global cardiovascular risk and other morbidity criteria. ${ }^{59} \mathrm{~A}$ particular focus should be given to patient assessment and adverse effects. ${ }^{60}$ Patient eligibility criteria for transfer (ie, patients with a confirmed diagnosis of diabetes) presenting few associated health problems should be validated in observational or experimental studies. ${ }^{39}$ Qualitative studies are also needed to assess the views of patients and other healthcare professionals that support or limit the acceptability of transfers. The importance of transferability of processes for many health problems other than diabetes is an argument for an integrative patient-centred care approach for task shifting. It may concern a larger group of patients with cardiovascular health problems and a large category of processes (ie, prevention and education) as implemented in GPs' practices or healthcare centres abroad. ${ }^{61}$ This global approach will require more coordination and shared decision making. ${ }^{35}$ Then, in a fragmented primary care system like that in France, it is necessary to further investigate the added value of a generic integrated model of coordinated care compared with single disease approaches. ${ }^{39}$

Economic aspects might favour a comprehensive teambased approach to patient care. Indeed, the efficacy of patient education and therapeutic counselling has been demonstrated $^{41}$ and some healthcare systems, such as the USA, even reward them financially through Current Procedural Terminology codes, ${ }^{38}{ }^{62}$ conversely to the French billing nomenclature. Education and counselling are regarded as non-existent in the healthcare databases and in French general practice even though, at a practical level, many GPs manage and initiate education for their patients. ${ }^{26336364}$ The modality of intervention and funding is different between hospital and GP practice encounters based on hospital-based activity, or is given either at a flat rate or on a per capita basis in the countries where it exists. ${ }^{64}$ Recognition through a specific billing code for education, equivalent for all professionals with adequate qualifications, would enable more time to be dedicated to these activities. ${ }^{64}$ Then, according to locally defined interprofessional collaborations, educational processes could be implemented by an increasing number of independent professionals with a minimum of integration (information system, protocols and supervision). More enhancement of such collaborations would probably require considering the team as a whole rather than the separate professions within the team, from both the organisational and financial perspectives. ${ }^{57}$ Irrespective of the approach, policymakers will have to allocate additional public funds to increase the acceptability of process of care transfer by key professionals. ${ }^{57}$ Therefore, the policy should be more oriented towards funding and enhancing new roles in new organisational models of primary care delivery rather than pushing to simplify the process of task transfer from one independent health professional to another. ${ }^{656}$

\section{CONCLUSIONS}

With regard to GPs in day-to-day practice in France, there is significant potential to transfer processes of care for patients with diabetes. Attention should be paid to the type of process to be transferred; this is the most important 
factor that determines the applicability of the process for transfer. Preventive processes, especially educational processes, are the most transferable, having a low risk-benefit ratio that favours education. Due to the frequency of associated cardiovascular risk factors, the transferability of educational processes should be large enough to address the global cardiovascular risk. Research questions on the professionals involved, their qualifications, remuneration, interprofessional education and organisational context should be further studied.

Acknowledgements We acknowledge the ECOGEN study group, including the steering committee, the residents, the $128 \mathrm{GP}$ trainers, the 54 residents. The members of the steering committee were: Laurent Letrilliart, Alain Mercier, Irène Supper, Matthieu Schuers, David Darmon, Pascal Boulet, Dominique Ambros, Madeleine Favre, Gil Mury, Bernard Gay, Denis Pouchain, Eric Van Ganse, Philippe Ameline, Anne-Marie Schott, Angelique Denis. The residents (observers) were: Céline Alexanian, Clement Barletta, Solene Baron de Preville, Muriel Baudoin-Bion, Naïma Belarbia, Clarisse Bertrand, Anne-Sophie Billet, Emilie Boulard, Emilie Breillat, Claire Brunet, Claire Camilleri, Hélène Carrier, Mathieu Carron, Nelly Cordeiro, Clément Coutarel, Sophie Dargent, Sarah Darriau, Hubert de Lary, Karen Denis, Yohana Dery, Isabelle Duquenne, Guillaume Farcis-Morgat, Charlotte Favier, Sarah Filoche, Mohamad Hamade, Marion Helly, Laura Hsiung, Thibault Lelong, Nathalie,Levernier, Julia Marquant, Prisca Martin, Caroline Martin-Bouyer, Ryma Metahri, Lesley-Ann Montigneaut, Noémie Morel, David Nakache, Claire Parker, Eric Pernollet, Solène Petitclerc, Alicia Pillot, Henri Plancke, Fanny Poirot, Thomas Proboeuf, Sophie Quien, Marie-Camille Rault-Tandonnet, Charlotte Regnier, Yohan Saynac, Saphanie Son, Damien Steciuk, Aurélie Urena-Dores, Yannick Vacher, Maxime Veques, Lucile Wies, Elodie Youssef. The participating GPs were: Ahmed Aadjour, Isabelle Aubin-Auger, Ghislaine Audran, Nadine Ayme, Catherine Bageot, Jérôme Bard, Bruno Beauchamps, Olivier Bisch, Paul Blanchet, Jean-Michel Blondel, Pierre Bobey, Jean-Yves Borgne, Jean-Yves Breton, Agnès Bryn, Martin Buisson, Marie Cabanas, Gérald Catsanedo, Maxime Cauchie, Nicole Caunes, Christine Cerisier-Cornillot, Patrick Charbit, Pascal Clerc, Laurent Convert, Françoise Corlieu, Thierry Cornille, Alain Couatarmanac'h, Claude Danner, Jean-Claude Darrieux, Alain Dasse, François de Golmard, Gilles de Lorenzi, Anto de Pavljasevic, Pierre-François Delzanno, Nicole Derain, Pierre Deveche, Vincent Diquero, Bénédicte Chevreau, Christian Larcheron, Elise Dubreuil, Pierre Dupont, Charline Dupont, Richard Dymny, Catherine Elsass, Pierre Eterstein, Gilles Faivre, Eric Fanjeaux, Emmanuelle Farcy, Claudine Fity, Vasantha Flory, Anne Girard, Christophe Girault, Sabine Grutter, Murielle Guillier, Thérèse Guyenne-Chambru, Christophe Haguet, Jean-Yves Hascoet, Sophie Haudidier, Sylvain Hirsch, Gaëtan Houdard, Hélène Hubail, André Kastelik, Sylvain Kichelewski, Xavier Lainé, Valérie Lapouge, David Laurent, Laurent Laval, Serge Lavaure, Mireille Lavigne, Yves Leborgne, Odile Lion, Viviane Mannevy, Jean-Michel Mathieu, Laure-Emmanuelle Mavraganis, Denis Perrot, Yvon Petrault, Christophe Pigache, Maurice Ponchant, Véronique Poupet, Daniel Reynolds, Emmanuel Robin, Marie-Hélène Robineau, Jean-Loup Roblot, Larisa Savan, Pierre Sebbag, Patrick Serey, Michel Serraille, Corinne Simoneau, François Tahon, Jean Louis Teruel, Audrey Tordoir, Christian Verot, Valérie Zéline. The French medical schools involved in ECOGEN study were : Amiens, Angers, Besançon, Bordeaux, Brest, Clermont Ferrand, Dijon, Grenoble, Lille (Etat), Limoges, Lyon, Marseille, Montpellier, Nancy, Nantes, Nice, Paris Descartes, Paris Pierre et Marie Curie, Paris Diderot, Paris Est Créteil, Paris Ile-de-France Ouest, Poitiers, Rennes, Rouen, St-Etienne, Strasbourg, Tours. The University College of General Practice of Lyon for the funding of the English editing.

\section{Collaborators The ECOGEN study group}

Contributors LL conceived the cross-sectional study. LL and IS designed the cross-sectional study. Interns of the ECOGEN study group collected the data. YB revised the protocol for data analysis. IS, $L L$ and RE analysed the data. IS wrote the first draft, which was revised by RE, YB and LL. IS, RE, YB and LL approved the final draft.

Funding This work was supported by the French National College of teachers in general practice and Pfizer.

\section{Competing interests None declared.}

Patient consent Detail has been removed from the case description(s) to ensure anonymity. The editors and reviewers have seen the detailed information available and are satisfied that the information backs up the case the authors are making.
Ethics approval National committee for informatics and freedom, CNIL $n^{\circ} 1549782$ and the committee for the protection of the persons, CPP $n^{\circ} \mathrm{L} 11-149$.

Provenance and peer review Not commissioned; externally peer reviewed.

Data sharing statement Technical appendix, statistical code, and dataset are not available from the Dryad repository. The dataset is available upon request from laurent.letrilliar@@univ-lyon1.fr. The corresponding author is available for further information.

Open Access This is an Open Access article distributed in accordance with the Creative Commons Attribution Non Commercial (CC BY-NC 4.0) license, which permits others to distribute, remix, adapt, build upon this work non-commercially, and license their derivative works on different terms, provided the original work is properly cited and the use is non-commercial. See: http://creativecommons.org/ licenses/by-nc/4.0/

(c) Article author(s) (or their employer(s) unless otherwise stated in the text of the article) 2017. All rights reserved. No commercial use is permitted unless otherwise expressly granted.

\section{REFERENCES}

1. Wild S, Roglic G, Green A, et al. Global prevalence of diabetes: estimates for the year 2000 and projections for 2030. Diabetes Care 2004;27:1047-53.

2. Bodenheimer T, Wagner EH, Grumbach K. Improving primary care for patients with chronic illness. JAMA 2002;288:1775-9.

3. Sarwar N, Gao P, Seshasai SR, et al. Diabetes mellitus, fasting blood glucose concentration, and risk of vascular disease: a collaborative meta-analysis of 102 prospective studies. Lancet 2010;375:2215-22.

4. Sibbald B, Shen J, McBride A. Changing the skill-mix of the health care workforce. J Health Serv Res Policy 2004;9(Suppl 1):28-38.

5. Samuelson M, Tedeschi P, Aarendonk D, et al. Improving interprofessional collaboration in primary care: position paper of the European Forum for Primary Care. Qual Prim Care 2012;20:303-12.

6. Laurant $\mathrm{M}$, Harmsen $\mathrm{M}$, Wollersheim $\mathrm{H}$, et al. The impact of nonphysician clinicians: do they improve the quality and costeffectiveness of health care services? Med Care Res Rev 2009;66:36S-89S.

7. Martínez-González NA, Tandjung R, Djalali S, et al. The impact of physician-nurse task shifting in primary care on the course of disease: a systematic review. Hum Resour Health 2015;13:55

8. Mousquès J, Bourgueil Y, Le Fur P, et al. Effect of a French experiment of team work between general practitioners and nurses on efficacy and cost of type 2 diabetes patients care. Health Policy 2010;98:131-43.

9. Cleveringa FG, Gorter KJ, van den Donk M, et al. Combined task delegation, computerized decision support, and feedback improve cardiovascular risk for type 2 diabetic patients: a cluster randomized trial in primary care. Diabetes Care 2008;31:2273-5.

10. Renders CM, Valk GD, Griffin S, et al. Interventions to improve the management of diabetes mellitus in primary care, outpatient and community settings. Cochrane Database Syst Rev 2001:CD001481.

11. Michot P, Catala O, Supper I, et al. [Cooperation between general practitioners and pharmacists: a systematic review]. Sante Publique 2013;25:331-41.

12. Tricco AC, Ivers NM, Grimshaw JM, et al. Effectiveness of quality improvement strategies on the management of diabetes: a systematic review and meta-analysis. Lancet 2012;379:2252-61.

13. Norris SL, Nichols PJ, Caspersen CJ, et al. The effectiveness of disease and case management for people with diabetes. A systematic review. Am J Prev Med 2002;22:15-38.

14. Amundson HA, Butcher MK, Gohdes D, et al. Montana cardiovascular disease and diabetes prevention program workgroup. Translating the diabetes prevention program Into practice in the general community. Diabetes Educ 2009;35:209-23.

15. Pariser G, Hager K, Gillette P, et al. Active steps for diabetes: a community-campus partnership addressing frailty and diabetes. Diabetes Educ 2014;40:60-7.

16. Baudier F, Bourgueil Y, Evrard I, et al. Group practice dynamics among private general practitioners from 1998 to 2009. QES 2010;157.

17. Tazim V. Interprofessional Collaborative Teams. Canadian Health Services Research Foundation, 2012.

18. Clément M-C, Couralet P-E, Mousquès J, et al. Les maisons de santé : un nouvel équilibre entre accessibilité, continuité des soins et organisation des médecins libéraux. Santé Publique 2009;21:79-90.

19. Struijs JN, Baan CA, Schellevis FG, et al. Comorbidity in patients with diabetes mellitus: impact on medical health care utilization. BMC Health Serv Res 2006;6:84. 
20. Teljeur C, Smith SM, Paul G, et al. Multimorbidity in a cohort of patients with type 2 diabetes. Eur J Gen Pract 2013;19:17-22.

21. Boeckxstaens P, Peersman W, Goubin G, et al. A practice-based analysis of combinations of diseases in patients aged 65 or older in primary care. BMC Fam Pract 2014;15:159.

22. Le Reste JY, Nabbe P, Manceau B, et al. The European General Practice Research Network presents a comprehensive definition of multimorbidity in family medicine and long term care, following a systematic review of relevant literature. J Am Med Dir Assoc 2013:14:319-25.

23. Boyd CM, Fortin M. Future of multimorbidity research: how should understanding of multimorbidity inform health system design? Public Health Rev 2010;32:451-74.

24. Valderas JM, Starfield B, Sibbald B, et al. Defining comorbidity: implications for understanding health and health services. Ann Fam Med 2009;7:357-63.

25. World Health Organization. World Health Report: Primary Health Care Now More Than Ever, 2008.

26. Letrilliart L, Supper I, Schuers M, et al. ECOGEN : étude des Éléments de la COnsultation en médecine GENérale. exercer 2014;114:148-57.

27. Okkes I, Jamoulle M, Lamberts $\mathrm{H}$, et al. ICPC-2-E: the electronic version of ICPC-2. Differences from the printed version and the consequences. Fam Pract 2000;17:101-7.

28. L'Institut national de la statistique et des études économiques. Nomenclature des professions et catégories socioprofessionnelles/ PCS. http://www.insee.fr/fr/methodes/default.asp?page= definitions/nomencl-prof-cat-socio-profes.htm (accessed 6 Sep 2016).

29. République française. Article $n^{\circ} 51$, Law $n^{\circ} 2009-879$ du 21 juillet 2009 portant réforme de l'hôpital et relative aux patients, à la santé et aux territoires. 2009https://www.legifrance.gouv.fr/affichTexte.do? cidTexte=JORFTEXT000020879475\&categorieLien=id (accessed 12 Oct 2017).

30. Letrilliart L, Rigault-Fossier P, Fossier B, et al. Comparison of French training and non-training general practices: a cross-sectional study. BMC Med Educ 2016;16:1-9.

31. Pillot A, Supper I, Guerin M-H, et al. Transférabilité des procédures de soins des médecins généralistes à d'autres professionnels de santé : une étude transversale nationale multicentrique. exercer 2014;114:186-93.

32. Jovic L, Bianchi E, Decouflet $\mathrm{S}$, et al. Nurses in France: between autonomy and subordination in front line care. Glob Qual Nurs Res 2015;2.

33. Agamaliyev E, Mikol F, Prost T. Les déterminants de l'opinion des médecins généralistes sur la délégation de tâches vers les infirmiers de leur cabinet. Rev Fr Aff Soc 2016;1:375-404.

34. Jaffiol C. [Current management of type 2 diabetes in France]. Bull Acad Natl Med 2009;193:1645-61.

35. Ogedegbe G, Gyamfi J, Plange-Rhule J, et al. Task shifting interventions for cardiovascular risk reduction in low-income and middle-income countries: a systematic review of randomised controlled trials. BMJ Open 2014;4:e005983.

36. Vargas RB, Mangione CM, Asch S, et al. Can a chronic care model collaborative reduce heart disease risk in patients with diabetes? $J$ Gen Intern Med 2007;22:215-22.

37. Echouffo-Tcheugui JB, Kengne AP. On the importance of global cardiovascular risk assessment in people with type 2 diabetes. Prim Care Diabetes 2013;7:95-102.

38. Ayyagari P, Grossman D, Sloan F. Education and health: evidence on adults with diabetes. Int J Health Care Finance Econ 2011;11:35-54.

39. Battersby MW. Health reform through coordinated care: SA HealthPlus. BMJ 2005;330:662-5.

40. Legifrance, le Service Public de la Diffusion du Droit.LOI ${ }^{\circ}$ 2016-41 du 26 janvier 2016 de modernisation de notre système de santé. 2017 https://www.legifrance.gouv.fr/eli/loi/2016/1/26/ AFSX1418355L/jo/texte.

41. Schellenberg ES, Dryden DM, Vandermeer B, et al. Lifestyle interventions for patients with and at risk for type 2 diabetes: a systematic review and meta-analysis. Ann Intern Med 2013;159:543-51.
42. Moreau A, Supper I. Effets des interventions éducatives sur la santé des patients diabétiques de type 2. exercer 2011:191-200.

43. Gaede P, Vedel P, Parving HH, et al. Intensified multifactorial intervention in patients with type 2 diabetes mellitus and microalbuminuria: the Steno type 2 randomised study. Lancet 1999;353:617-22.

44. Knowler WC, Barrett-Connor E, Fowler SE, et al. Reduction in the incidence of type 2 diabetes with lifestyle intervention or metformin. N Engl J Med 2002;346:393-403.

45. Boussageon R, Supper I, Bejan-Angoulvant T, et al. Reappraisal of metformin efficacy in the treatment of type 2 diabetes: a meta-analysis of randomised controlled trials. PLoS Med 2012;9:e1001204.

46. Erpeldinger S, Rehman MB, Berkhout C, et al. Efficacy and safety of insulin in type 2 diabetes: meta-analysis of randomised controlled trials. BMC Endocr Disord 2016;16:39.

47. Monami M, Genovese S, Mannucci E. Cardiovascular safety of sulfonylureas: a meta-analysis of randomized clinical trials. Diabetes Obes Metab 2013;15:938-53.

48. Tierney $\mathrm{K}$. Therapeutic choices, and the nurse practitioner's role, in type 2 diabetes when metformin alone is no longer enough. $J \mathrm{Am}$ Acad Nurse Pract 2012;24 Suppl 1:234-48.

49. Courtenay M, Carey N, Gage H, et al. A comparison of prescribing and non-prescribing nurses in the management of people with diabetes. J Adv Nurs 2015;71:2950-64.

50. Houweling ST, Kleefstra N, van Hateren KJ, et al. Can diabetes management be safely transferred to practice nurses in a primary care setting? A randomised controlled trial. $J$ Clin Nurs 2011;20:1264-72.

51. Stange KC. The problem of fragmentation and the need for integrative solutions. Ann Fam Med 2009;7:100-3.

52. May C, Montori VM, Mair FS. We need minimally disruptive medicine. BMJ 2009;339:b2803.

53. Haggerty JL, Reid RJ, Freeman GK, et al. Continuity of care: a multidisciplinary review. BMJ 2003;327:1219-21.

54. Starfield B, Shi L, Macinko J. Contribution of primary care to health systems and health. Milbank Q 2005;83:457-502.

55. Chomienne MH, Grenier J, Gaboury I, et al. Family doctors and psychologists working together: doctors' and patients' perspectives. J Eval Clin Pract 2011;17:282-7.

56. Winefield HR, Turnbull DA, Seiboth C, et al. Evaluating a program of psychological interventions in primary health care: consumer distress, disability and service usage. Aust N Z J Public Health 2007;31:264-9.

57. Supper I, Catala O, Lustman M, et al. Interprofessional collaboration in primary health care: a review of facilitators and barriers perceived by involved actors. J Public Health 2015;37:716-27.

58. Laurant M, Reeves D, Hermens R, et al. Substitution of doctors by nurses in primary care. Cochrane Database Syst Rev 2005:CD001271.

59. Hansen LJ, Drivsholm TB. [Interventions to improve the management of diabetes mellitus in primary health care and outpatient community settings]. Ugeskr Laeger 2002;164:607-9.

60. Starfield B. Politics, primary healthcare and health: was Virchow right? J Epidemiol Community Health 2011;65:653-5.

61. Marek $\mathrm{A}$, Mousquès $\mathrm{J}$. The participation of nurses in primary care in six European countries, Ontario and Quebec. Issues Health Econ IRDES 2005;95.

62. Dickey RA. Practical tips on coding for diabetes care. Endocr Pract 1996;2:389-94.

63. Trombert-Paviot B, Rector A, Baud R, et al. The development of CCAM: the new French coding system of clinical procedures. HIM J 2003;31:2-11.

64. Haut Conseil de Santé Publique. L'éducation thérapeutique intégrée aux soins de premier recours. 2009.

65. Bourgueil Y. Skill-mix reform in France. Patients, peers, professionals: Skill-mix innovationsand developments in primary and chronic care settings in Europe: WHO Eur Obs, 2017.

66. Bourgueil Y, Marek A, Mousquès J. Three Models of Primary Care Organisation in Europe, Canada, Australia and New-Zealand. Issues Health Econ IRDES 2009;141. 